\title{
Téoros
}

Revue de recherche en tourisme

\section{Un début de millénaire turbulent}

\section{Michèle Laliberté}

Volume 22, numéro 3, automne 2003

URI : https://id.erudit.org/iderudit/1071349ar

DOI : https://doi.org/10.7202/1071349ar

Aller au sommaire du numéro

\section{Éditeur(s)}

Université du Québec à Montréal

\section{ISSN}

0712-8657 (imprimé)

1923-2705 (numérique)

Découvrir la revue

Citer cette note

Laliberté, M. (2003). Un début de millénaire turbulent. Téoros, 22(3), 55-56. https://doi.org/10.7202/1071349ar d'utilisation que vous pouvez consulter en ligne.

https://apropos.erudit.org/fr/usagers/politique-dutilisation/ 


\section{Chaire de tourisme}

\section{Un début de millénaire turbulent}

\section{Michèle Laliberté}

J amais n'a-t-on vu une telle concentration d'événements venir perturber l'industrie touristique en un si court laps de temps. Bien que chaque partie du globe ait été bousculée, les conséquences ont pris différentes tangentes selon la situation géographique et l'espace temps ${ }^{1}$. L'industrie touristique québécoise n'a pas échappé à cette vague de répercussions et en a subi, parfois indirectement, les contrecoups.

\section{Un bilan des événements}

La demande touristique mondiale des dernières années se profile en dents de scie (OMT, 2003) :

2001

- Croissance relativement faible de la demande (2,9\%) pour les mois de janvier à août en raison de l'incertitude économique.

- Chute de 8,6 \% pour la période de septembre à décembre pour finalement se solder par un fléchissement des arrivées de l'ordre de $0,5 \%$ (684,1 millions d'arrivées de touristes internationaux) par rapport à l'année précédente.

2002

- Malgré une fin d'année marquée par l'attentat de Bali et la menace de la guerre en Irak, augmentation de la demande de $2,7 \%$ (702,6 millions).

2003

- Performances plutôt inégales dans chaque région en raison de la guerre en Irak, du SRAS et d'un climat économique toujours instable.

- Impact immédiat du SRAS sur la demande touristique en Asie et au Canada, affectant aussi les régions qui transigent avec ces marchés.

- Dès juillet, observation d'une lente reprise sur les marchés les plus touchés.

- Croissance de 0 à $3 \%$ prévue par l'Organisation mondiale du tourisme (OMT).

Globalement, les événements du 11 septembre 2001 ont un effet désastreux sur la demande ; le SRAS se révèle plus néfaste que la guerre en Irak et le contexte économique incertain, en fil de trame, en freine l'accroissement. La demande se caractérise par une contraction du tourisme international vers le tourisme régional et intérieur et certaines tendances déjà présentes s'accentuent accentuées, notamment les réservations de dernière minute, le tourisme de proximité et les courts séjours.

En raison de leur faible sensibilité au prix, les voyageurs d'affaires constituent depuis longtemps la manne de l'industrie touristique. Or, au cours des dernières années, on assiste à une certaine mutation. Les entreprises resserrent leur politique de dépenses ; le facteur « prix » devenant une préoccupation, une part grandissante de voyageurs d'affaires utilise les transporteurs à tarifs réduits et effectue ses réservations en ligne. Quant à la clientèle d'agrément, les prévisions de croissance ont été longtemps perçues comme prometteuses notamment en raison de certains facteurs démographiques. Cependant, la crainte des attentats terroristes, les mesures de sécurité renforcées, les frais élevés et les taxes de toutes sortes, de même que l'importante baisse de performance des fonds de pension constituent autant d'éléments qui pourraient entraîner une révision de la courbe de croissance envisagée.

\section{Les gagnants et les perdants}

Le bilan économique des récents événements totalise plusieurs milliards de dollars de pertes pour l'industrie touristique. Le continent nord-américain sort grand perdant de cette bataille, ayant été assailli de toutes parts. L'Asie-Pacifique s'en tirait plutôt bien jusqu'à l'arrivée du SRAS, tandis que l'Europe, ayant adopté une attitude de «wait and see » et en raison de ses liens avec les principaux marchés touchés, a subi, elle aussi, les contrecoups de ces événements.

En tête de liste des secteurs d'activités les plus touchés, les transporteurs aériens internationaux ont de la difficulté à reprendre leur souffle. Au lendemain du 11 septembre, les titres boursiers des compagnies aériennes américaines effectuent une importante plongée. Déjà fragilisées, certaines compagnies aériennes sont contraintes de fermer leurs portes. Premier transporteur aérien au monde, American Airlines évite la faillite de justesse tandis que d'autres se trouvent sous la protection de la loi de la faillite et qu'un grand nombre accuse de lourdes pertes financières. L'Organisation de l'aviation civile internationale estime des pertes nettes globales s'élevant à plus de 30 milliards 
de dollars américains pour les années 2001 à 2003. Dans un tel contexte, les compagnies aériennes réduisent leur nombre de vols et leur capacité, suppriment jusqu'à ce jour plus de 400000 emplois (IATA, 2003), négocient de nouvelles ententes salariales avec les syndicats et diminuent leurs coûts d'exploitation. Bien que la reprise semble amorcée et ne soit pas uniforme dans toutes les régions, le secteur aérien montre un optimisme prudent et plusieurs analystes ne prévoient un recouvrement significatif de la demande qu'en 2005. Par ailleurs, même si la demande se mettait à progresser rapidement, cela ne signifie pas que la rentabilité sera au rendez-vous.

En raison d'importantes pertes financières, les voyagistes se concentrent à la consolidation de leur entreprise et ralentissent leur mouvement d'intégration verticale. Quant aux agences de voyages, plusieurs ferment leurs portes. Leur positionnement face au mouvement sans cesse croissant de la distribution en ligne, de l'émergence du dynamic packaging et des coupures des commissions les confrontent à d'importants défis. Dans le domaine de l'hébergement, les hôtels de classe intermédiaire et économique se tirent le mieux d'affaires, ce qui s'explique notamment par la sensibilité au prix de la part de la clientèle d'affaires. De même, les hôteliers indépendants affichent une meilleure feuille de route que les grandes chaînes.

Alors que les dernières années sont plutôt éprouvantes pour l'ensemble de l'industrie touristique, certains secteurs tirent toutefois profit du contexte. Ainsi, dans le secteur aérien, on constate que la grisaille n'est pas le tribut de tous les acteurs. En effet, plusieurs transporteurs à tarifs réduits alignent des chiffres de croissance impressionnants ${ }^{2}$, en raison notamment de leur pénétration significative du marché des voyageurs d'affaires et de l'effervescence du marché intérieur. Dans la même foulée, le secteur du voyage en ligne résiste à la tourmente. Les résultats des années 2001 et 2002 traduisent un accroissement remarquable des réservations en ligne avec des hausses respectives de 59 et de 37 \% (PhoCusWright, 2002), le contexte défavorable ayant principalement entraîné une diminution de l'inventaire en transport aérien pour les agences.

\section{Un regard sur l'industrie touristique québécoise}

L'onde de choc des événements des dernières années se répercute évidemment sur le Québec. Alors que la sécurité définissait la logique du voyageur et que ce dernier se tournait vers le tourisme de proximité, cette réalité se traduit pour le Québec par une baisse de la clientèle internationale et une appréhension du voyageur québécois qui, autrement, aurait visité une destination étrangère. Les régions qui attirent une grande part de la clientèle hors-frontière sont les plus durement touchées, notamment Montréal, Québec et Mont-Tremblant. En période de croissance, le secteur touristique courtise davantage la clientèle internationale, boudant quelque peu le marché intérieur, mais, lorsque surviennent des menaces géopolitiques ou autres, les campagnes marketing se replient sur le dernier. On n'a qu'à se rappeler, à l'hiver suivant les événements du 11 septembre, la campagne de la Commission canadienne du tou- risme vantant les beautés du Canada. Jamais les Canadiens n'avaient été courtisés d'une façon aussi convaincante. Par contre, depuis plusieurs années, Tourisme Québec s'est engagé sur cette voie en faisant la promotion de ses régions de façon assidue.

Avec la reprise qui se profile, la concurrence entre les destinations sera d'autant plus vive, chaque destination voulant redorer son image et regagner le terrain perdu. Quels seront les futurs gagnants, quels seront les perdants ? Chose certaine, la donne sera changée. À cet effet, la dernière tourmente nous oblige à réfléchir sur notre vulnérabilité, l'évolution de notre offre et de sa qualité, notre image et notre positionnement par rapport aux autres destinations. Nos visées internationales se révèlent légitimes, mais s'avèrent toutefois ambitieuses. En mode « réaction » depuis plusieurs années et souvent décalée dans ses actions par rapport aux événements, l'industrie touristique québécoise est confrontée à une image quelque peu défraîchie. L'effervescence de l'Exposition universelle de 1967 et des Jeux olympiques de 1976 étant chose du passé, il en reste des infrastructures vieillissantes et un vide en matière de projets novateurs qui pourraient rehausser la qualité de l'offre et l'image de la destination.

Que retenir de cette succession de perturbations? Plus que jamais, nous avons besoin de réduire notre temps de réaction en période de crise, de renouveler le produit, de mettre en valeur et de favoriser les régions à fort potentiel touristique, de s'inspirer des actions les plus novatrices afin de répondre aux attentes de demain. Pour ce faire, une obligation de veille intelligente s'impose pour mieux comprendre, comparer et orienter.

\section{Michèle Laliberté est professionnelle de recherche au Réseau} de veille en tourisme.

\section{Notes}

1 Ce texte s'inspire du document de réflexion présenté par la Chaire de tourisme lors de la $15^{\mathrm{e}}$ Assemblée générale de l'Organisation mondiale du tourisme tenue à Beijing en octobre 2003 - L'industrie du tourisme : un modèle d'affaires à réinventer.

2 Aux États-Unis, Southwest jouit d'une capitalisation boursière qui dépasse les six plus grands transporteurs américains réunis ; en 2002, il occupait le sixième rang du classement de tous les transporteurs mondiaux sur le plan des profits nets. Au Canada, malgré le contexte difficile des dernières années, WestJet affiche une rentabilité pour un $26^{\mathrm{e}}$ trimestre consécutif. En matière de marge d'exploitation, en Europe, Ryanair (Irlande) occupe le premier rang de tous les transporteurs - à tarifs réduits et réguliers -, avec $31 \%$.

\section{Bibliographie}

IATA - International Air Transport Association (2003), Industry in Crisis, Change Essential, 2 juin.

Organisation mondiale du tourisme (2003), Tourism Highlights Edition 2003, octobre.

PhoCusWright Inc. (2002), « Online Travel Market Update 20012005 », TRAVstats, 30 septembre. 